\title{
Electrorheological Fluid Dynamics
}

\author{
Jianwei Zhang, ${ }^{1,3}$ Xiuqing Gong, ${ }^{1}$ Chun Liu, ${ }^{2,3}$ Weijia Wen, ${ }^{1}$ and Ping Sheng ${ }^{1,3}$ \\ ${ }^{1}$ Department of Physics, The Hong Kong University of Science and Technology, Clear Water Bay, Kowloon, Hong Kong, China \\ ${ }^{2}$ Department of Mathematics, Pennsylvania State University, State College, Pennsylvania 16802, USA \\ ${ }^{3}$ Kavli Institute for Theoretical Physics China, Chinese Academy of Sciences, Beijing 100190, China
}

(Received 11 June 2008; published 5 November 2008)

\begin{abstract}
We use the Onsager principle to derive a two-phase continuum formulation for the hydrodynamics of the electrorheological (ER) fluid, consisting of dielectric microspheres dispersed in an insulating liquid. Predictions of the theory are in excellent agreement with the experiments. In particular, it is shown that whereas the usual configuration of applied electric field being perpendicular to the shearing direction can lead to shear thinning at high shear rates and thus the loss of ER effect, the interdigitated, alternating electrodes configuration can eliminate the shear-thinning effect.
\end{abstract}

DOI: 10.1103/PhysRevLett.101.194503

PACS numbers: 47.65.Gx, 47.50.-d, 83.50.Ax, 83.80.Gv

Electrorhoelogical (ER) fluids [1-11], consisting of solid particles dispersed in an insulating carrier fluid, constitute a class of colloids whose rheological characteristics are controllable through the application of an external electric field. They have broad applications potential in active dampers, valves, etc. [2,3]. While the static ER characteristics have been studied successfully with the effective dielectric constant formulation [1,6,11], the dynamic behavior of ER fluids still represents a challenging area. Direct simulations involving a number of discrete, electrically interacting particles would be computationally limited by the particle number [12-18]. Bingham fluid [19] has often been used for the prediction of ER dynamics, in which the dynamic shear stress $\tau$ is given by $\tau=\tau_{0}+\eta \dot{\gamma}$, with $\eta$ being the viscosity, $\dot{\gamma}$ the shear rate, and $\tau_{0}$ the threshold shear stress beyond which the fluidlike behavior is recovered. While the Bingham model clearly captures an essential element of the ER dynamics, it fails to account for the often-observed shear-thinning behavior and the sensitivity of ER rheology to electrode configuration(s).

In this Letter, we present a two-phase continuum model for the ER fluid dynamics, in which the electrical interaction energy between the solid particles is treated on the basis of (induced) dipole-dipole interaction, valid in the limit of weak ER effect. By regarding the number density of solid particles as a field variable, we derive the equations of motion by using the Onsager principle of least dissipation [20-22]. Results obtained are in excellent agreement with the experiments. In particular, it is shown that the shear-thinning behavior of ER dynamics may be avoided by using a planar, alternate-electrode configuration. This can have positive implications for ER fluid applications.

Consider solid microspheres of radius $a$, dielectric constant $\varepsilon_{s}$, and mass $m$ suspended in oil with dielectric constant $\varepsilon_{f}$. In the presence of external field $\vec{E}^{\text {ext }}$, the solid particles will be polarized with an induced dipole moment $\vec{p}=\left[\left(\varepsilon_{s}-\varepsilon_{f}\right) /\left(\varepsilon_{s}+2 \varepsilon_{f}\right)\right] a^{3} \vec{E}^{\ell}$, where $\vec{E}^{\ell}$ denotes the local electric field. Interaction between the induced dipoles means a phase separation into two components. Below, we first focus on the dense colloidal phase, denoted the " $s$ " component.

To regularize the interaction energy, we assume the point dipole $\vec{p}$ to be situated at the center of the microsphere, and a repulsive interaction potential is introduced between any two spheres $i$ and $j$ (situated at $\vec{x}$ and $\vec{y}$, respectively) as $\chi(|\vec{x}-\vec{y}|)=\varepsilon_{o}(a /|\vec{x}-\vec{y}|)^{12}$, where $\varepsilon_{o}$ is a suitably chosen energy constant. We treat the solid particles collectively by regarding their density $n(\vec{x})=$ $f_{s}(\vec{x})\left(4 \pi a^{3} / 3\right)^{-1}$ as a field variable, where $f_{s}(\vec{x})$ denotes the local volume fraction of microspheres. The viscosity of the dense colloidal phase, which is affected by the particleparticle repulsive interaction, will be modeled as a function of $n$, fitted to experimental results. Free energy for the $s$ component may be expressed as

$$
\begin{aligned}
F[n(\vec{x})]= & -\frac{1}{2} \int G_{i j}(\vec{x}, \vec{y}) p_{i}(\vec{x}) n(\vec{x}) p_{j}(\vec{y}) n(\vec{y}) d \vec{x} d \vec{y} \\
& -\int \vec{E}^{\operatorname{ext}}(\vec{x}) \cdot \vec{p}(\vec{x}) n(\vec{x}) d \vec{x} \\
& +\frac{1}{2} \int \chi(|\vec{x}-\vec{y}|) n(\vec{x}) n(\vec{y}) d \vec{x} d \vec{y}
\end{aligned}
$$

where $G_{i j}(\vec{x}, \vec{y})=-\nabla_{i} \nabla_{j}|\vec{x}-\vec{y}|^{-1}$ is the dipole-dipole interaction operator, and repeated indices imply summation in Eq. (1). We ignore the entropic effects because they are much smaller than the electrical energies of the relevant particle sizes under consideration. A variation of $F$ [23-27] with respect to $n$ leads to $\delta F=\int \mu[n(\vec{x})] \delta n d \vec{x}$, where $\mu[n(\vec{x})]=-\vec{E}^{\ell}(\vec{x}) \cdot \vec{p}(\vec{x})+\int \chi(|\vec{x}-\vec{y}|) n(\vec{y}) d \vec{y} \quad$ is $\quad$ the chemical potential, with $E_{i}^{\ell}(\vec{x})=E_{i}^{\operatorname{ext}}(\vec{x})+$ $\int G_{i j}(\vec{x}, \vec{y}) p_{j}(\vec{y}) n(\vec{y}) d \vec{y}$ denoting the local field. Since $n$ is locally conserved, there is a continuity equation for $n$, given by $\dot{n}+\vec{\nabla} \cdot \vec{J}=\partial_{t} n+V_{s} \cdot \vec{\nabla} n+\vec{\nabla} \cdot \vec{J}=0$, where 
$V_{s}$ is the " $"$ " phase velocity, and $\vec{J}$ is a convective-diffusive current density. It is now desirable to derive the expression for $\vec{J}$, as well as the coupled equations of motion for the two components, via the Onsager principle.

The Onsager action functional [20-22] is given by $A=$ $\dot{F}+\Phi$, where $\dot{F}$ is the time variation of the free energy and $\Phi$ is half the dissipation rate(s). The minimization of $A$ with respect to the rate(s) would lead to the equation(s) of motion which insures the balance between the dissipative forces and conservative forces (those derivable from free energy), as well as to the most probable dynamics of the dissipative processes. For the " $s$ " component, we have $A\left(\vec{J}, \vec{V}_{s}\right)=\dot{F}+\Phi$, where

$$
\begin{aligned}
\dot{F} & =\int \mu \partial_{t} n d \vec{x}=\int \mu\left(\dot{n}-\vec{V}_{s} \cdot \vec{\nabla} n\right) d \vec{x} \\
& =\left(\vec{\nabla} \mu \cdot \vec{J}+n \vec{\nabla} \mu \cdot \vec{V}_{s}\right) d \vec{x},
\end{aligned}
$$

and

$$
\begin{aligned}
\Phi= & \int\left(\frac{1}{4} \eta_{s}\left[\partial_{i}\left(\vec{V}_{s}\right)_{j}+\partial_{j}\left(\vec{V}_{s}\right)_{i}\right]^{2}\right. \\
& \left.+\frac{\gamma}{2 n} J^{2}+\frac{1}{2} K\left(\vec{V}_{f}-\vec{V}_{s}\right)^{2}\right) d \vec{x},
\end{aligned}
$$

together with the incompressibility condition $\vec{\nabla} \cdot \vec{V}_{s}=0$, which can be implemented by using a Lagrange multiplier $\lambda$. In Eq. (3), $\gamma$ is a frictional coefficient. Energy dissipation rate per unit volume is $n \gamma V_{d}^{2}=\gamma J^{2} / n$. Taking into account the factor $1 / 2$ leads directly to the expression shown in Eq. (3). The other two terms of $\Phi$ are simply the well-known viscous dissipation, with $\eta_{s}$ denoting the colloidal viscosity, and the dissipation caused by the friction between the two components, characterized by a constant $K$. We choose the lowest-order estimate of $K=$ $\gamma n$ that is linear in $n$, since the results show that $\vec{V}_{s} \sim \vec{V}_{f}$ so that the calculated stresses are insensitive to $K$. Variational minimization of the Onsager functional with respect to the rates $\left(\vec{J}, \vec{V}_{s}\right)$ leads to $\vec{J}=-(n / \gamma) \vec{\nabla} \mu$ and the Stokes equation

$$
0=-\vec{\nabla} p_{s}+\eta_{s} \nabla^{2} V_{s}+n \vec{\nabla} \mu+K\left(V_{f}-V_{s}\right),
$$

where $\lambda=-2 p_{s}$, and we propose the Stokes form $\gamma=$ $6 \pi \eta_{s} a$ for the friction coefficient. For the colloidal viscosity $\eta_{s}$, we have used numerical interpolation to reproduce the experimentally observed dependence $\eta_{s} / \eta_{f} \propto \exp \left[0.6 /\left(0.698-f_{s}\right)\right]$ at close to the random closed packing fraction of 0.698 [28], and to the form $\eta_{s} / \eta_{f}=1+2.5 f_{s}+O\left(f_{s}^{2}\right)$ at low solid particles concentration [29]. A similar but simpler calculation involving $\vec{V}_{f}$ would lead to the equation

$$
0=-\vec{\nabla} p_{f}+\eta_{f} \nabla^{2} V_{f}+K\left(V_{s}-V_{f}\right)
$$

for the fluid component. When the inertial effects are not negligible, momentum balance requires the left-hand sides of Eqs. (4) and (5) be replaced by $\rho_{s} \dot{\vec{V}}_{s}$ and $\rho_{f} \dot{\vec{V}}_{f}$, respectively, with $\rho_{s}=m n(\vec{x})+(1-f) \rho_{f}$. Together with the nonslip boundary conditions for both components, the above formulation forms a consistent mathematical scheme.

We have implemented the numerical codes [30] so as to make predictions that can be compared with the experiments. In Fig. 1(a), we show that for an electric field
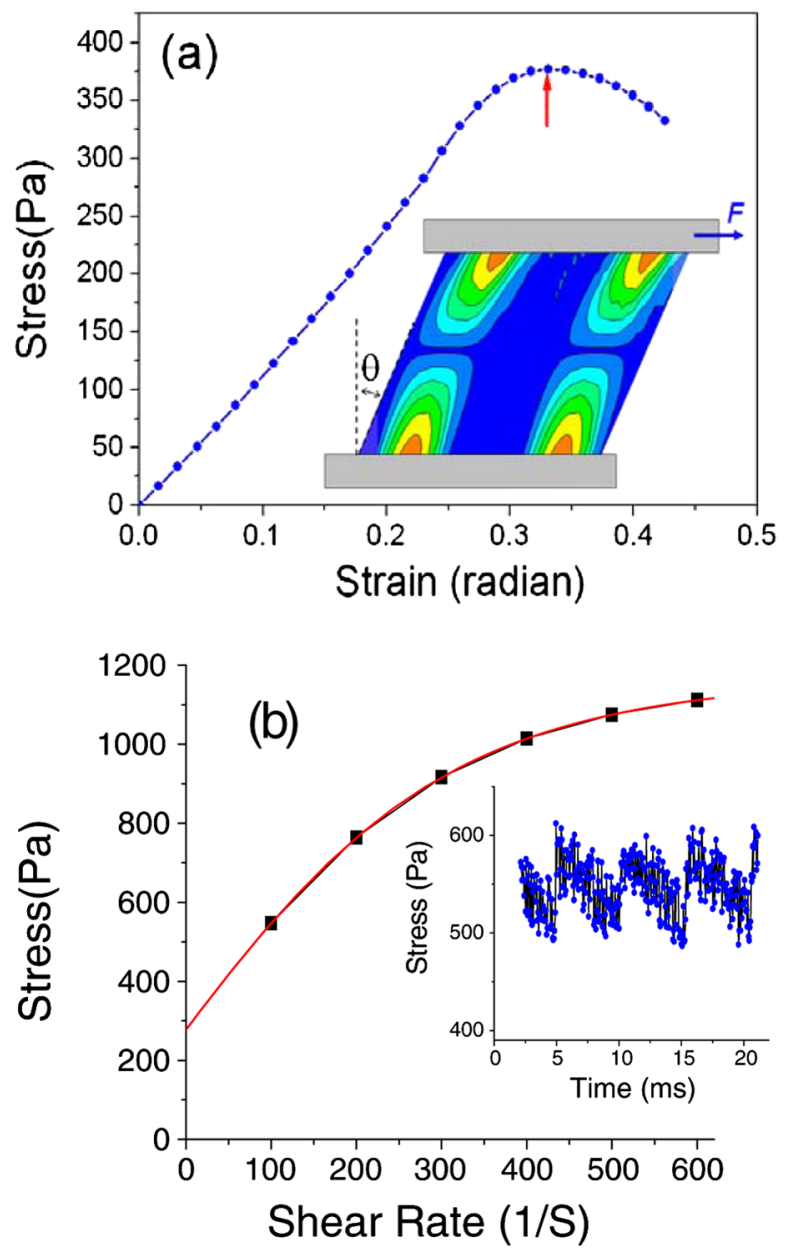

FIG. 1 (color online). (a) Calculated shear stress plotted as a function of strain (the angle $\theta$ ) under an electric field of $2 \mathrm{kV} / \mathrm{mm}$. The cell is $650 \mu \mathrm{m} \times 650 \mu \mathrm{m} \times 2 a$ ( $y$ direction), with periodic boundary condition along the shearing direction $x$. To facilitate the formation of columns under an electric field, the initial density is given by $n_{o}+\delta n \cos (k x)$. The inset shows the breaking of the columns at around the yield stress point. The static yield stress is $374 \mathrm{~Pa}$ in this case. (b) Calculated (averaged) dynamic shear stress under the Couette flow condition for the same cell as in (a). By extrapolating (with the solid line fitted to the simulation results) to zero shear rate, the dynamic yield stress is found to be $278 \mathrm{~Pa}$. The inset shows the stress fluctuations at a shear rate of $100 \mathrm{~s}^{-1}$. Here, $a=5 \mu \mathrm{m}, m=1.2 \times 10^{-9} \mathrm{~g}$, $\varepsilon_{s}=10, \varepsilon_{f}=2, \eta_{f}=10 \mathrm{cP}, \rho_{f}=0.96 \mathrm{~g} / \mathrm{cm}^{3}$, and overall $f_{s}=30 \%$. The zero-field shear stress is very small; hence, the behavior shown can be taken to be that for the ER effect only. 


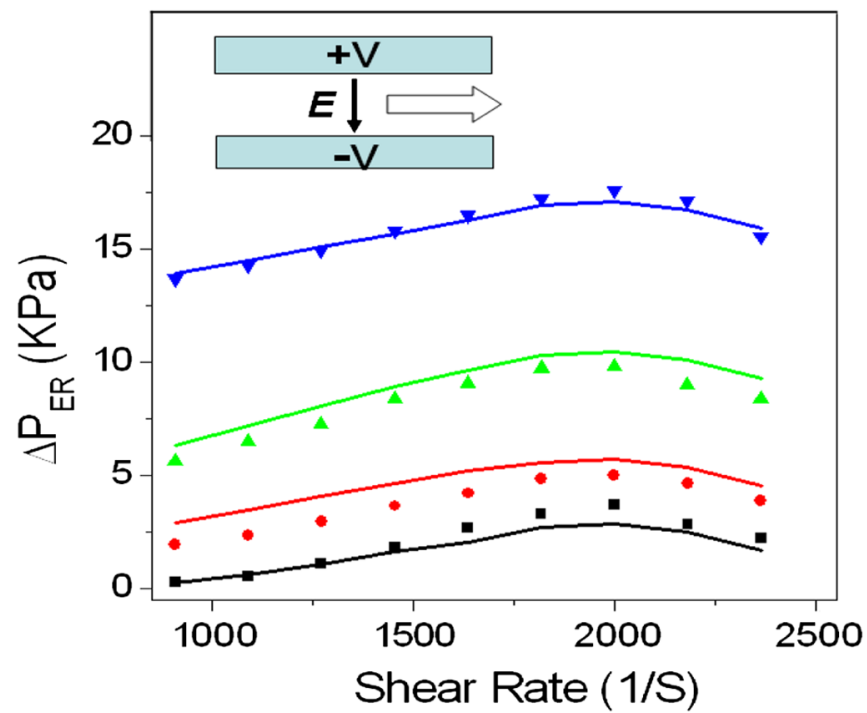

FIG. 2 (color online). The (time-averaged) pressure difference due to the ER effect $\Delta P_{\mathrm{ER}}=\Delta P_{\text {meas }}-\Delta P_{\text {visc }}$, plotted as a function of shear rate for the electrode configuration (with a gap of $1 \mathrm{~mm}$ ) shown in the inset. The symbols and lines represent the experimental and our theoretical results, respectively. From bottom to top: applied electric field is $1 \mathrm{kV} / \mathrm{mm}$, $2 \mathrm{kV} / \mathrm{mm}, 3 \mathrm{kV} / \mathrm{mm}$, and $4 \mathrm{kV} / \mathrm{mm}$. At $1 \mathrm{kV} / \mathrm{mm}$, the pressure difference is very small at low shear rates. Here, $a=$ $2.5 \mu \mathrm{m}, m=1.2 \times 10^{-10} \mathrm{~g}, \varepsilon_{s}=2.9, \varepsilon_{f}=2, \eta_{f}=50 \mathrm{cP}$, $\rho_{f}=0.96 \mathrm{~g} / \mathrm{cm}^{3}$, and overall $f_{s}=11.5 \%$.

applied across two parallel electrodes, the model can reproduce the ER shear elastic behavior up to a critical strain associated with the static yield stress, beyond which the fluid behavior emerges. The shear elasticity is the result of column formation as seen in the inset to Fig. 1(a). Here, red (dark) indicates a high value of $n$ and blue (light) as a low value. When the top plate is moved at a constant speed relative to the bottom plate to generate a Couette flow, the resulting shear stress experienced on the top plate is plotted as a function of time in the inset to Fig. 1(b). Fluctuations are seen which reflect the breaking and re-attachment of the columns. The time-averaged stress is plotted as a function of shear rate in Fig. 1(b). The behavior is very similar to the Bingham fluid at low shear rates, with an extrapolated dynamic yield stress that is $\sim 30 \%$ lower than the static yield stress shown in Fig. 1(a).

Experiments were done in the Poiseuille flow configuration, with different electrode configurations (see insets to Figs. 2 and 3). The ER fluid was prepared by dispersing molecular sieve particles (product type: $3 \mathrm{~A} 1 / 16,5 \mu \mathrm{m}$ in diameter, provided by Nacalai Tesque Inc., Japan) into the silicone oil with a particle concentration of $11.5 \mathrm{vol} . \%$. The prepared ER fluid was baked at $120^{\circ} \mathrm{C}$ for $1 \mathrm{~h}$ to remove any moisture. Tensile machine (MTS SINTECH 10/D Frame Specification) was used for the ER effect measurements, carried out with flow rates varying from $0.05-150 \mathrm{~mm} / \mathrm{min}$ through a constriction formed by two

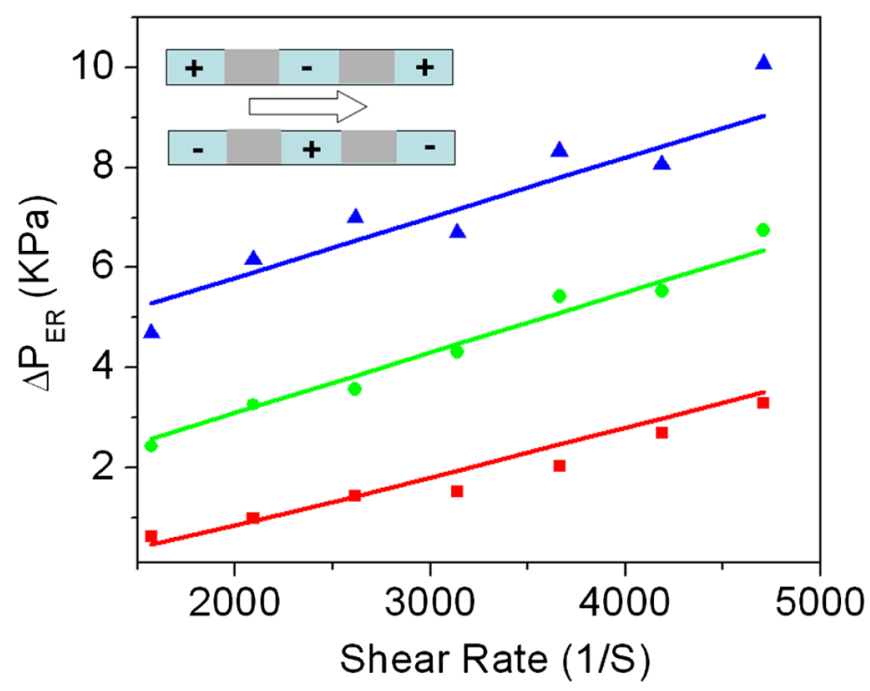

FIG. 3 (color online). The pressure difference due to the ER effect $\Delta P_{\mathrm{ER}}=\Delta P_{\text {meas }}-\Delta P_{\text {visc }}$, plotted as a function of shear rate for the planar, alternate-electrode configuration. The symbols and lines represent the experimental and our theoretical results, respectively. From bottom to top are electrical field equal to $1 \mathrm{kV} / \mathrm{mm}, 1.5 \mathrm{kV} / \mathrm{mm}$, and $2 \mathrm{kV} / \mathrm{mm}$. The parameter values used in the calculations are the same as that in Fig. 2.

parallel plates with a width of $1 \mathrm{~cm}$, length $4 \mathrm{~cm}$ and separated by $1 \mathrm{~mm}$ [31]. The force on the piston of the cell was measured by a force gauge and recorded with a software package. The resulting pressure difference on two ends of the constriction can be easily obtained from the time-averaged force. A DC power supply (SPELLMAN SL300) provided high voltages applied to the ER fluids.

In Fig. 2, it is seen that for electric field applied across the two parallel plates, there is clearly a shear-thinning behavior at high shear rates $[32,33]$. There is a simple explanation to this phenomenon based on the fact that the strength of the columns is always along the applied electric field. Hence, when the columns are tilted significantly away from the perpendicular field direction, the resistance to shear is decreased. The solid lines are the theory predictions. It is seen that the agreement is excellent. As the theoretical yield stress follows a strictly $E^{2}$ variation, the experimental results are seen to be in general agreement with this trend.

An alternative design involving the use of interdigitated electrodes (inset to Fig. 3) would mean that the applied electric field can have a significant component parallel to the shearing direction. Figure 3 shows the measured (symbols) and calculated (solid lines) results, up to a high shear rate of $4700 / \mathrm{s}$. The shear-thinning effect no longer occurs, seen to be correctly predicted by our continuum model with no adjustable parameters.

We wish to thank T.Z. Qian for helpful discussions and to acknowledge Hong Kong RGC Research Grant No. HKUST 604205 for the financial support of this work. 
[1] H. R. Ma, W. J. Wen, W. Y. Tam, and P. Sheng, Adv. Phys. 52, 343 (2003).

[2] M. Whittle and W. Bullough, Nature (London) 358, 373 (1992).

[3] T. Halsey, Science 258, 761 (1992).

[4] H. Block and J. P. Kelly, J. Phys. D 21, 1661 (1988).

[5] R. Tao and J. M. Sun, Phys. Rev. Lett. 67, 398 (1991).

[6] H. R. Ma, W. J. Wen, W. Y. Tam, and P. Sheng, Phys. Rev. Lett. 77, 2499 (1996).

[7] W. Y. Tam, G. H. Yi, W. Wen, H. R. Ma, M. M. T. Loy, and P. Sheng, Phys. Rev. Lett. 78, 2987 (1997).

[8] W. J. Wen, X. Huang, S. Yang, K. Lu, and P. Sheng, Nature Mater. 2, 727 (2003).

[9] W. Wen, X. Huang, and P. Sheng, Appl. Phys. Lett. 85, 299 (2004).

[10] X. Huang, W. Wen, S. Yang, and P. Sheng, Solid State Commun. 139, 581 (2006).

[11] W. Wen, X. Huang, and P. Sheng, Soft Matter 4, 200 (2008).

[12] R. T. Bonnecaze and J.F. Brady, J. Rheol. (N.Y.) 36, 73 (1992).

[13] R. T. Bonnecaze and J. F. Brady, J. Chem. Phys. 96, 2183 (1992).

[14] D. J. Klingenberg, F. V. Swol, and C. F. Zukoski, J. Chem. Phys. 91, 7888 (1989).

[15] D. J. Klingenberg, F. V. Swol, and C. F. Zukoski, J. Chem. Phys. 94, 6160 (1991).

[16] D. J. Klingenberg, J. Rheol. (N.Y.) 37, 199 (1993).

[17] D. J. Klingenberg, C. F. Zukoski, and J. C. Hill, J. Appl. Phys. 73, 4644 (1993).

[18] K. C. Hass, Phys. Rev. E 47, 3362 (1993).

[19] A. H. Skelland, Non-Newtonian Flow and Heat Transfer (John Wiley, NewYork, 1967).

[20] L. Onsager, Phys. Rev. 37, 405 (1931); Phys. Rev. 38, 2265 (1931).

[21] L. Onsager and S. Machlup, Phys. Rev. 91, 1505 (1953).

[22] L.D. Landau and E. M. Lifshitz, Statistical Physics (Addison-Wesley Publishing Co., London, 1969), 2nd ed..
[23] R. Ryham, C. Liu, and L. Zikatanov, Discrete and Continuous Dynamical Systems, Series B 8, 649 (2007).

[24] F. Lin, C. Liu, and P. Zhang, Commun. Pure Appl. Math. 60, 838 (2007).

[25] R. Ryham, Ph.D. thesis, Penn State University, 2006.

[26] J. J. Feng, C. Liu, J. Shen, and P. Yue, in Modeling of Soft Matter, IMA Volumes in Mathematics and its Applications (Springer, New York, 2005), Vol. 141, p. 1.

[27] C. Liu, J. Shen, J. J. Feng, and P. Yue, Mathematical Models and Methods in Phase Transitions (Nova Publishers, New York, 2005), Chap. 11.

[28] Z. Cheng, J. Zhu, P. Chaikin, S. Phan, and W. Russell, Phys. Rev. E 65, 041405 (2002).

[29] R. Verberg, I. de Schepper, and E. Cohen, Phys. Rev. E 55, 3143 (1997).

[30] The electric field configuration is obtained by solving the Laplace equation $\vec{\nabla} \cdot \bar{\varepsilon} \vec{\nabla} \phi=0$, where $\phi$ denotes the electrical potential, and the effective dielectric constant $\bar{\varepsilon}$ is locally updated by using the Maxwell-Garnett relation $\left[\bar{\varepsilon}(\vec{x})-\varepsilon_{f}\right] /\left[\bar{\varepsilon}(\vec{x})+2 \varepsilon_{f}\right]=f_{s}(\vec{x})\left(\varepsilon_{s}-\varepsilon_{f}\right) /\left(\varepsilon_{s}+2 \varepsilon_{f}\right)$. Consistency between the local field and $\vec{p}$ is obtained iteratively. In the numerical calculations, $n$ is taken to be $2 \mathrm{D}$, with periodic condition along $x$ (the shearing direction) and the thickness along the $y$ direction taken to be $2 a$, i.e., one layer of ER fluid.

[31] The ER fluid transit time through the electrode region is $0.04 \mathrm{~s}$ at the shear rate of $1000 \mathrm{~s}^{-1}$. This is $\sim 20$ times smaller than the $\tau=0.8 \mathrm{sec}$ set by the conductivity of the ER particles.

[32] From the experimental flow rate, the shear rate is evaluated as $D^{-1} \int_{0}^{D}|\partial V(z) / \partial z| d z$, where $D$ is the distance between the two electrodes and $V(z)$ the calculated velocity profile that matches the flow rate.

[33] Shear thinning is a result of balance of flow-induced forces against electrostatic interaction. Our results are qualitatively consistent with the theories presented in Ref. [3] and in J. Martin, J. Odinek, T. Halsey, and R. Kamien, Phys. Rev. E 57, 756 (1998). 